\title{
Magnetic Localization for Perching UAVs on Powerlines
}

\author{
Joseph Moore and Russ Tedrake
}

\begin{abstract}
Perching on powerlines to recharge provides a unique opportunity to extend the mission duration capabilities of small-scale UAVs (Unmanned Aerial Vehicles). In this paper, we investigate the feasibility of localizing an aircraft using the magnetic field generated by a current carrying wire through state estimation and hardware development. By using an Extended Kalman Filter to track the real and imaginary components of the magnetic field signal, we overcome the problems posed by the field's phase-amplitude ambiguity and demonstrate the ability to track an aircraft flying at speeds up to $8 \mathrm{~m} / \mathrm{s}$ at a distance of 4 meters from the wire. We conclude that the achieved performance is adequate for controlling a bird-scale UAV in a dynamic perching maneuver and that our system would generalize to real world scenarios.
\end{abstract}

\section{INTRODUCTION}

In recent years, there has been significant interest in small and micro UAVs, since they have demonstrated the potential to enable a number of new mission capabilities, including navigating in and around buildings, flying through forests, and performing perch-and-stare surveillance. However, these small UAVs possess a significant drawback when being used in the field- they are simply unable to carry sufficient power for long missions. For this reason, developing ways for these aircraft to increase their mission duration capabilities is absolutely crucial.

One approach to increasing UAV mission duration is by using energy that already exists in the environment to recharge, or power the UAV. Some of the initiatives that have pursued this line of research have focused on methods such as gust soaring [19], while others have sought to use solar panels to boost the flight capabilities of their aircraft[14], [4]. In urban areas, powerlines provide a unique opportunity for harvesting energy from the environment either through inductive coupling or direct connection. In the past few years, some successful research initiatives have resulted in technologies which allow soldiers to efficiently acquire electricity from existing power grids[6]. If a small UAV could successfully land on a powerline, it could potentially recharge using this technology while also conducting perch and stare surveillance. Furthermore, the fact that powerlines already generate a magnetic beacon makes them particularly advantageous, since such magnetic fields can be easily detected by autonomous aircraft.

In this paper, we address the challenge of localizing a perching aircraft using a powerline's magnetic field. This poses a number of fundamental challenges and questions. First, there is a question of signal strength, since the field strength drops off as $\frac{1}{r^{3}}$, where $r$ is the distance from the line. Second, because the onboard sensor does not know the absolute phase of the current in the wire, the inverse model

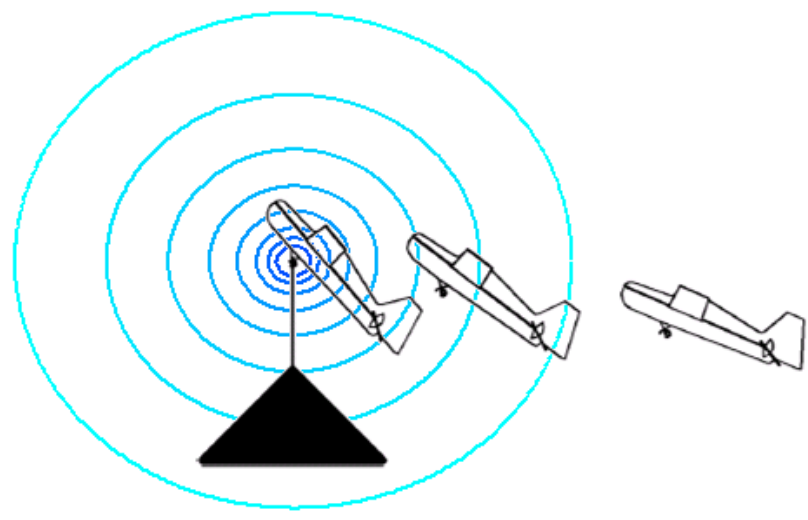

Fig. 1. Powerline Perching

has ambiguity - there are many potential locations relative to the perch that can give the same instantaneous measurements. Our approach to overcome these problems is to use an aircraft model and state estimation to resolve this ambiguity by fusing measurements from a three-axis magnetometer and an onboard IMU. To obtain performance which is robust to process model errors, we develop a measurement model to track the the real and imaginary components of a magnetic field signal and, as a result, disambiguate the field. Using this technique, we are able to achieve accurate localization on a UAV moving up to $8 \mathrm{~m} / \mathrm{s}$ up to $4 \mathrm{~m}$ away from the powerline.

In the following sections, we review some previous work and the basics of powerline class and configuration. Then we provide a brief overview of the magnetic field model and signal processing algorithms; these were initially presented in [10], but have been improved in order to obtain the results we present here. Next we present the state estimation algorithm and implementation with onboard hardware. Finally we present the results of on-board tracking of the post-stall perching trajectory, and conclude with a discussion of the applicability of these results to more general scenarios.

\section{RELATED WORK}

From the early days of space exploration, measurements of the earth's magnetic field have been crucial to satellite attitude estimation and control[16], [18], [13]. In recent years, UAVs have also begun to make use of the earth's magnetic field to obtain heading measurements[9]. In addition to using the earth's magnetic field, the robotics community has made use of magnetic beacons to localize and control mobile robots. In [17], the author uses magnetic dipoles to achieve collision avoidance between multiple UAVs. Similarly, in [12], the author uses multiple Extremely Low Frequency 
(ELF) magnetic field beacons to localize a mobile robot in a factory setting. Although no work other than [10] has been done to use the magnetic fields generated by powerlines for estimation, some work has recently been done to model powerline magnetic fields for health purposes. In [5], a flexible code is created to compute the magnetic field around arbitrary conductor configurations. In [8], the impacts of sagging electrical conductors are explored by using the BiotSavart law to generate a more accurate magnetic model.

With regards to fixed-wing perching, a number of research efforts have been undertaken in the past few years. In [20] and [21], researchers attempt to achieve fixed wing perching by the use of a platform with morphing wings. By rotating the wings down during the perching maneuver, their aircraft was able to maintain attached flow while using the drag of the pitched fuselage to slow down the vehicle. [3] and [15] have taken a different approach to the perching problem by seeking to embrace the full nonlinear dynamics to control a perching aircraft at very high angles of attack.

In many ways, perching on a powerline to recharge is a natural extension of the fixed-wing perching maneuvers explored in [3] and [15]. Such a technological advancement would be a significant step towards developing far-ranging fixed-wing aircraft which could recharge autonomously in the field.

\section{Powerline Class and Configuration}

Before beginning any analysis, it is important to identify the class and configuration of the powerline which will be used in the experiments.

\section{A. Powerline Voltage Class}

In most power distribution grids, the powerlines can be divided into three main categories based on voltage. After electricity is generated by the power plant, it is stepped up to extremely high voltages. These high voltage lines, also known as transmission lines, carry electricity long distances at voltages from 69-765 $\mathrm{kV}$ [1]. These transmission lines typically run into a power substation where the voltage levels are stepped down. The first step down results in voltages between 34.5 and $69 \mathrm{kV}$, which are known as sub-transmission lines. Eventually, the subtransmission line voltages are stepped down again to distribution voltage levels, and carried on what are known as distribution lines [1]. Since the distribution lines carry the greatest currentsfor instance, a $10 \mathrm{kV}$ distribution line serving a $1 \mathrm{MW}$ load will produce 100 Amps of current- these are the lines which would seem to be the most feasible for inductive recharging.

\section{B. Conductor Configuration}

Today, most of the world uses three-phase power systems. These polyphase systems require three conductors to carry alternating currents of the same frequency but offset by 120 degrees in phase[2]. Although most primary distribution lines reflect this configuration, secondary distribution lines will often only consist of one or two of these three phases.

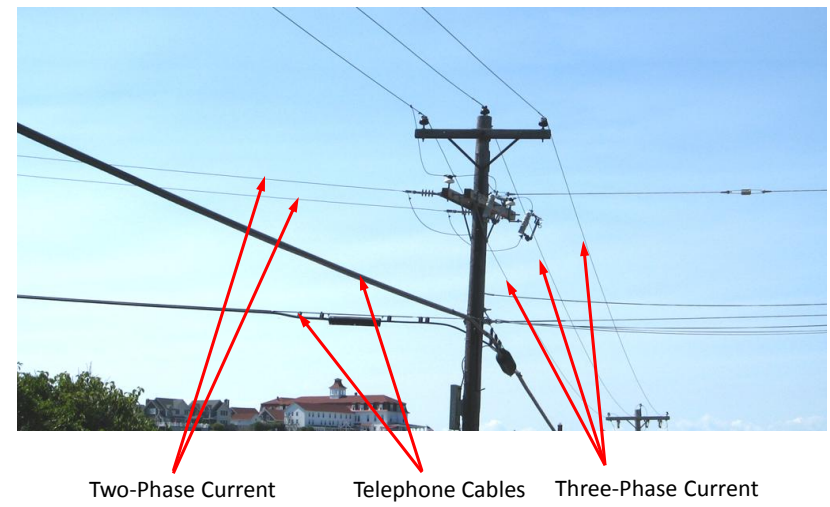

Fig. 2. Three-Phase to Single Phase Powerline Configuration

At first glance, it seemed as though an experimental setup using a single conductor carrying a current of a single phase would be the best choice. Not only would such a configuration reflect some of the existing distribution lines already in use, but it would also allow for the simplest magnetic field model to be developed. However, this setup poses some difficulty, for it would require the magnetic shielding of the return path - an extremely difficult task at low frequencies. To avoid this, the next best configuration, a rectangular current loop, was chosen.

\section{Magnetic Field Modeling}

To localize an aircraft in a magnetic field using a state estimator, one needs a measurement model, that is, a model representing the magnetic field measurements as seen by the sensor. In [10], this model was derived for a set infinite parallel wires as seen in Figure 3 using Ampere's Law. However, this model proved to be inadequate, since it did not take into consideration the full rectangular loop configuration. To

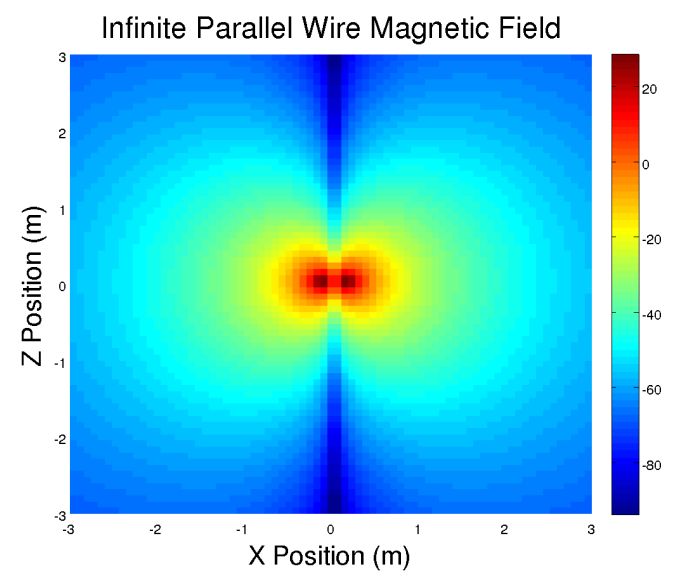

Fig. 3. Dipole Magnetic Field

model the rectangular current loop, the Biot-Savart law was applied as shown in Figures 4 and 5, since this law allows one to compute the magnetic field generated by an infinitesimal section of wire. The Biot-Savart Law can be formulated as, 


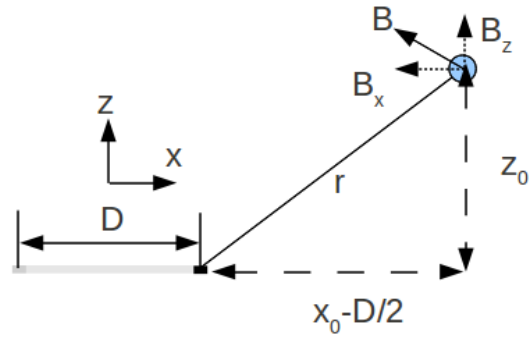

Fig. 4. Side View of Rectangular Current Loop

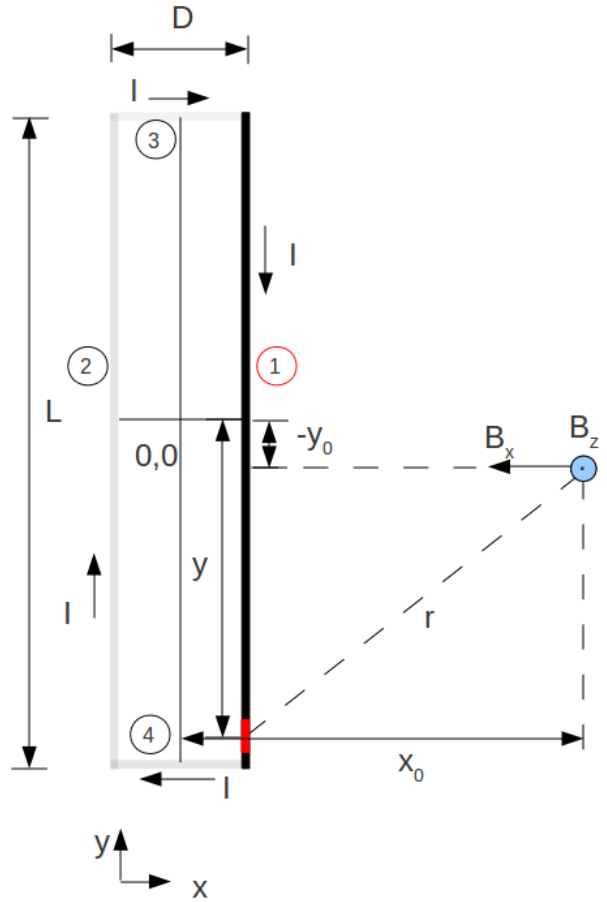

Fig. 5. Top View of Rectangular Current Loop

$$
\mathbf{B}=\int \frac{\mu_{0} I d \mathbf{l} \times \mathbf{r}}{4 \pi r^{3}}
$$

where $\mu_{0}$ is the magnetic permeability of free space, $I$ is the current in the wire, $l$ is the vector from the origin of the wire to the infinitesimal wire segment of interest in the direction of the current, and $\mathbf{r}$ is the vector from the infinitesimal wire segment to the aircraft location. In the case of the first wire, as shown in Figure 5, these terms can be rewritten as,

$$
\begin{aligned}
\mathbf{l} & =-y \hat{\mathbf{j}} \\
d \mathbf{l} & =-d y \hat{\mathbf{j}} \\
\mathbf{r} & =\left(x_{0}-\frac{D}{2}\right) \hat{\mathbf{i}}+\left(y_{0}-y\right) \hat{\mathbf{j}}+z_{0} \hat{\mathbf{k}}
\end{aligned}
$$

where $x_{0}, y_{0}$, and $z_{0}$ define the position of the aircraft, and $\hat{\mathbf{i}}, \hat{\mathbf{j}}$, and $\hat{\mathbf{k}}$ represent the Cartesian unit vectors. Taking the cross product between $d \mathbf{l}$ and $\mathbf{r}$ yields,

$$
d \mathbf{l} \times \mathbf{r}=\left(-z_{0} \hat{\mathbf{i}}+\left(x_{0}-\frac{D}{2}\right) \hat{\mathbf{k}}\right) d y
$$

Then, substituting into the Biot-Savart Integral gives,

$$
\mathbf{B}_{\mathbf{1}}=\int_{-\frac{L}{2}}^{\frac{L}{2}} \frac{\mu_{0} I\left(-z_{0} \hat{\mathbf{i}}+\left(x_{0}-\frac{D}{2}\right) \hat{\mathbf{k}}\right) d y}{4 \pi\left(\sqrt{\left(x_{0}-\frac{D}{2}\right)^{2}+\left(y_{0}-y\right)^{2}+z_{0}^{2}}\right)^{3}}
$$

which is a vector containing the $x$ and $z$ components of the magnetic field, $B_{x, 1}$ and $B_{x, 2}$, as a function of position from first wire. In the same way, three other integrals can be evaluated to obtain $B_{y, 3}, B_{y, 4}, B_{z, 1}, B_{z, 2}, B_{z, 3}$ and $B_{z, 4}$, which are the vector field contributions from the three remaining wires. The full magnetic field equations then become

$$
\begin{aligned}
B_{x} & =B_{x, 1}+B_{x, 2} \\
B_{y} & =B_{y, 3}+B_{y, 4} \\
B_{z}=B_{z, 1}+B_{z, 2} & +B_{z, 3}+B_{z, 4}
\end{aligned}
$$

Last of all, we must take into consideration the rotation of the sensor, where $R_{\phi}$ is the rotation matrix representing roll, $R_{\theta}$ represents pitch, and $R_{\psi}$ represents yaw.

$$
\mathbf{B}^{\prime}=R_{\phi} R_{\theta} R_{\psi} \mathbf{B}
$$

$\mathbf{B}^{\prime}$ will then serve as our measurement model.

\section{Signal Processing}

Because the magnetic field signal generated by the powerline is $\mathrm{AC}$ in nature, one can use conventional signal processing methods to obtain the magnitude and phase of the signal. One such method is complex synchronous modulation.

\section{A. Complex Synchronous Demodulation}

It is well known that any sinusoidal signal with a given phase can be decomposed into both a cosine component and sine component[11].

Furthermore, we know that the modulated message signal, $y_{m}$, is equivalent to,

$$
y_{m}(t)=m(t) \cos \left(\omega_{c} t+\phi\right)
$$

where $\omega_{c}$ is the carrier frequency in $\mathrm{rad} / \mathrm{s}, t$ is time, $m$ is the message signal, and $\phi$ is the signal phase.

When $y_{m}$ is multiplied by $\sin \omega t$ and $\cos \omega t$, the result yields

$$
\begin{aligned}
& y_{m, c}(t)=m(t) \frac{\cos \left(2 \omega_{c} t+\phi\right)+\cos (\phi)}{2} \\
& y_{m, s}(t)=m(t) \frac{\sin \left(2 \omega_{c} t+\phi\right)+\sin (\phi)}{2}
\end{aligned}
$$

After these signals are low-pass filtered, the results are

$$
\begin{aligned}
& y_{m, c}(t)=m(t) \frac{\cos (\phi)}{2} \\
& y_{m, s}(t)=m(t) \frac{\sin (\phi)}{2}
\end{aligned}
$$

Notice that these two signal are 90 degrees out of phase with one another and together they represent the real and complex components of the message signal. 


\section{Phase-Amplitude Ambiguity}

Although complex synchronous demodulation performs extremely well at recovering the magnitude of the message signal, it can not distinguish between a 180 degree phase offset and a negative field magnitude. This occurs because knowledge of the powerline's absolute phase does not exist on-board the aircraft. This feature can create a significant tracking difficulty, especially if only the signal magnitudes are used. When combined with the magnetic field model, such a phenomenon creates eight positions where the aircraft will obtain identical measurements. Phase-amplitude ambiguity is a major challenge facing state estimation using the AC magnetic field generated by a powerline. A diagram of the phase ambiguity can be seen in Figure 6.

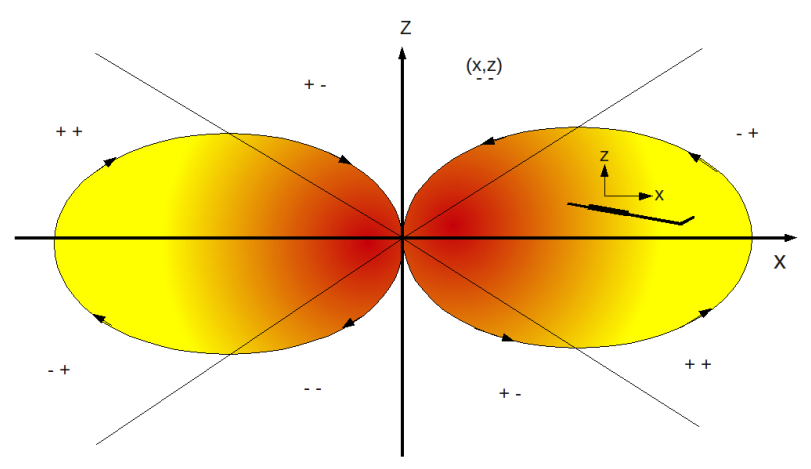

Fig. 6. Phase Ambiguity Diagram

\section{STATE Estimation}

Typically state estimation trades off a measurement model and a process model to achieve a desirable tracking performance. During the course of this investigation, several state estimation methods were explored, including particle filters, unscented Kalman filters, and Extended Kalman Filters. All three state estimation algorithms used the model of the magnetic field as seen by the sensor as the measurement model. For the process model, both an aircraft model based on flatplate theory and a double integrator were used. In the end, it was determined that the extended Kalman filter provided the best performance given the required computational cost.

\section{A. Extended Kalman Filter}

The extended Kalman filter (EKF) is a nonlinear form of the classical Kalman filter, which computes the observer gains by linearizing about the current state estimate. The EKF algorithm is as follows:

Compute the forward plant dynamics using the nonlinear model,

$$
\hat{x}_{t \mid t-1}=f\left(\hat{x}_{t-1}, u_{t}\right)
$$

where $\hat{x}$ is the system state, $t$ is time, $f$ represents a nonlinear function, and $u_{t}$ is the system input. Then, determine the error covariance matrix as well as the Kalman gains in the following manner:

Compute the a priori error covariance matrix as,

$$
P_{t \mid t-1}=F_{t} P_{t} F_{t}^{T}+Q_{t}
$$

where $Q_{t}$ is the process covariance matrix and $F_{t}$ represents the process gradients. The observer gain matrix $K_{t}$ can the be computed as,

$$
K_{t}=P_{t \mid t-1} H_{t}^{T}\left[H_{t} P_{t \mid t-1} H_{t}^{T}+R_{t}\right]^{-1}
$$

where, $R_{t}$ is the measurement covariance and $H_{t}$ represents the measurement gradients. Then compute the a posteriori error covariance matrix,

$$
P_{t}=\left(I-K_{t} H_{t}\right) P_{t \mid t-1}
$$

Finally, update the state estimate by multiplying Kalman gain matrix by the measurement error as shown,

$$
\hat{x}_{t}=\hat{x}_{t \mid t-1}+K_{t}\left(y_{t}-y\left(\hat{x}_{t \mid t-1}\right)\right)
$$

where $y$ is the output of the measurement model and $y_{t}$ is the actual measurement at time $t$.

\section{B. Complex Signal Tracking}

To incorporate the real and imaginary components of the magnetometer signal into the measurement model, the magnetic field model can be written as,

$$
\begin{aligned}
B_{x, c} & =B_{x} \cos (\phi) \\
B_{x, s} & =B_{x} \sin (\phi) \\
B_{z, c} & =B_{z} \cos (\phi) \\
B_{z, s} & =B_{z} \sin (\phi) \\
\phi & =m t+b
\end{aligned}
$$

where $\phi$ is the phase, $m$ and $b$ are unknown constants, and $t$ is time.

\section{System ARCHITECTURE}

The overall system architecture can be seen in Figure 7.

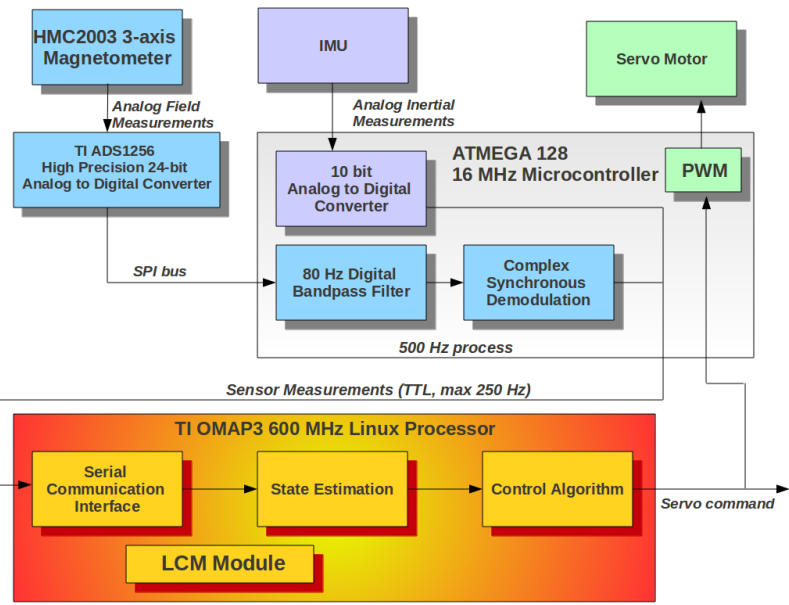

Fig. 7. System Block Diagram 


\section{A. Hardware Design}

To achieve powerline perching, a significant effort was undertaken to develop hardware capable of sensing lowlevel magnetic fields and providing reliable state estimation at high update rates. The GOSHAWK magnetic sensing system, which can be seen in Figures 8 and 9, emerged as a low-cost, light-weight solution. The GOSHAWK mag-

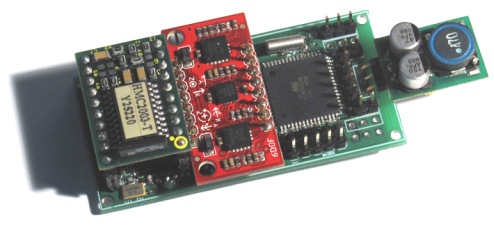

Fig. 8. Top-View of GOSHAWK Sensing Board

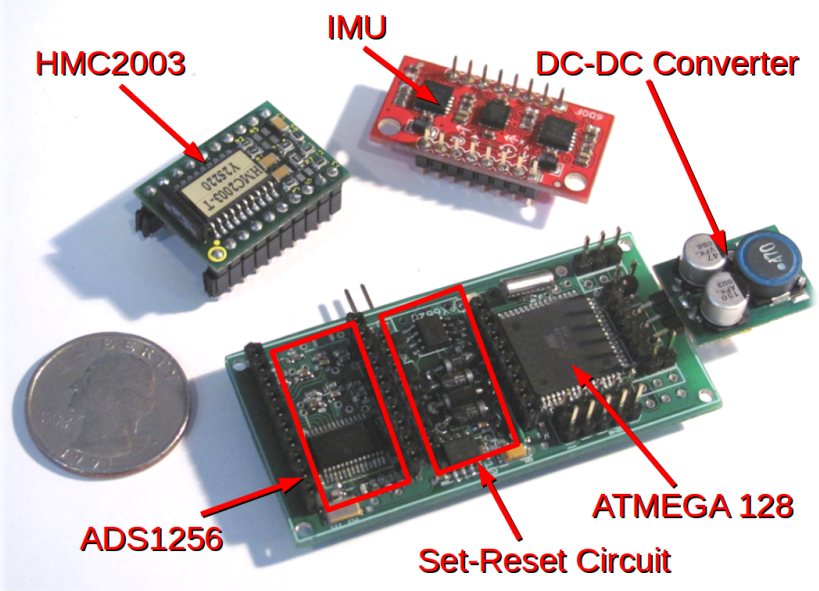

Fig. 9. Exploded-View of GOSHAWK Sensing Board

netic sensing system consists of a Honeywell magnetometer (HMC2003), a 24-bit high fidelity analog-to-digital converter (ADS1256), an ATMEGA128 microcontroller for low-level signal processing, a Gumstix Overo motherboard for high level computation, and an IMU for inertial measurement. The Gumstix Overo motherboard, which is not shown here, communicates with the GOSHAWK Sensing Board over a serial port. Together, the sensing board and Gumstix Overo motherboard weigh less than 35 grams and are able to measure magnetic field signals down to a few hundred microGauss with reasonable precision. Including batteries, the entire instrumented aircraft weighs around 115 grams.

\section{B. Low-level Signal Processing}

Most of the low-level signal processing occurs on the ATMEGA128 microcontroller. It is here that the magnetometer signals are sampled and undergo the complex synchronous demodulation process previously described. In addition to the complex synchronous demodulation algorithm, a bandpass filter is used to attenuate high frequencies and the magnetometer DC bias before demodulation occurs. A notch filter is also used to remove the $60 \mathrm{~Hz}$ noise, since $60 \mathrm{~Hz}$ is fairly close in frequency to the $80 \mathrm{hz}$ carrier frequency.

It is important to note that when implementing the digital signal processing on board the microcontroller it is necessary to sample at least $4 \mathrm{x}$ the carrier frequency in order to prevent the residual $2 \omega_{c}$ terms from aliasing down.

When the digital signal processing algorithms were implemented in hardware, the magnetic field magnitude measured exhibited a standard deviation of about $30 \mu \mathrm{G}$.

\section{High Level State-Estimation and Control}

Both of the computationally intensive state-estimation and control are located as $\mathrm{C}$ programs on the Gumstix Overo processor. These programs receive magnetic field and IMU data from the microcontroller at speeds up to $340 \mathrm{~Hz}$, and use that data to control the aircraft during a perching maneuver. To communicate between processes and to stream data over wireless, Lightweight Communications and Marshalling [7] was used.

\section{EXPERIMENTAL SET-UP}

To implement the rectangular current loop, 4 gage welding wire was used to carry a peak current near 40 amps. This current level was selected to provide just about four times the minimum field strength capable of being picked up by the magnetic sensors $(40 \mu \mathrm{V})$ at 4 meters from the wire. To generate the AC current, a PWM motor amplifier was used. This amplifier was fed by a 600 watt DC power supply and driven by a conventional signal generator to adjust the frequency of the current. To create the dipole configuration, a wooden stand was built to support a rectangular loop of wire 2.4 meters long by 0.3 meters wide. Furthermore, the wire leads bringing current to and from the power electronics were arranged in a twisted pair configuration so as to ensure that the only magnetic field being generated by the wire would only be due to the loop. Figure 10 shows a block diagram of the experimental powerline set up.

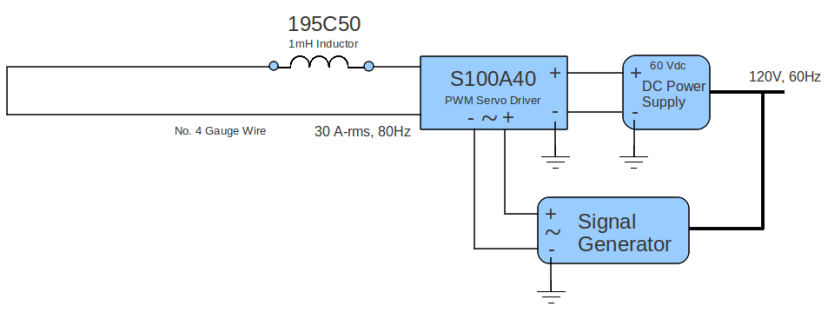

Fig. 10. Rectangular Current Loop Block Diagram

\section{Results}

\section{A. Simulation}

Initially, the Extended Kalman Filter was applied using only the absolute value of the magnetic field signal and an aircraft model with very low process noise. Even in throwing away the phase information, tracking was shown to be successful in simulation as displayed in Figure 11. 


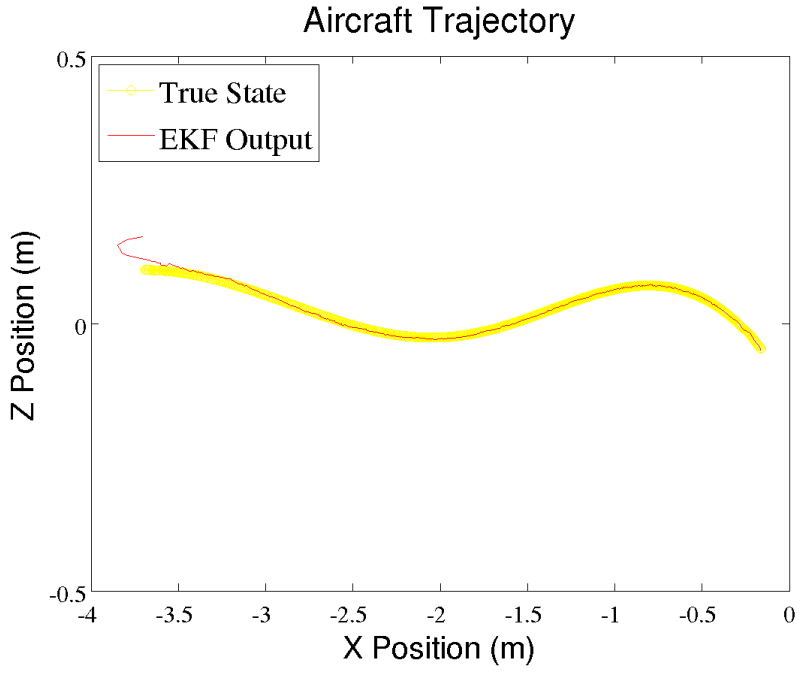

Fig. 11. Extended Kalman Filter Simulation

However, as the model was changed to a simple double integrator and the process noise was increased, the Extended Kalman Filter failed, as shown in Figure 12. It is important

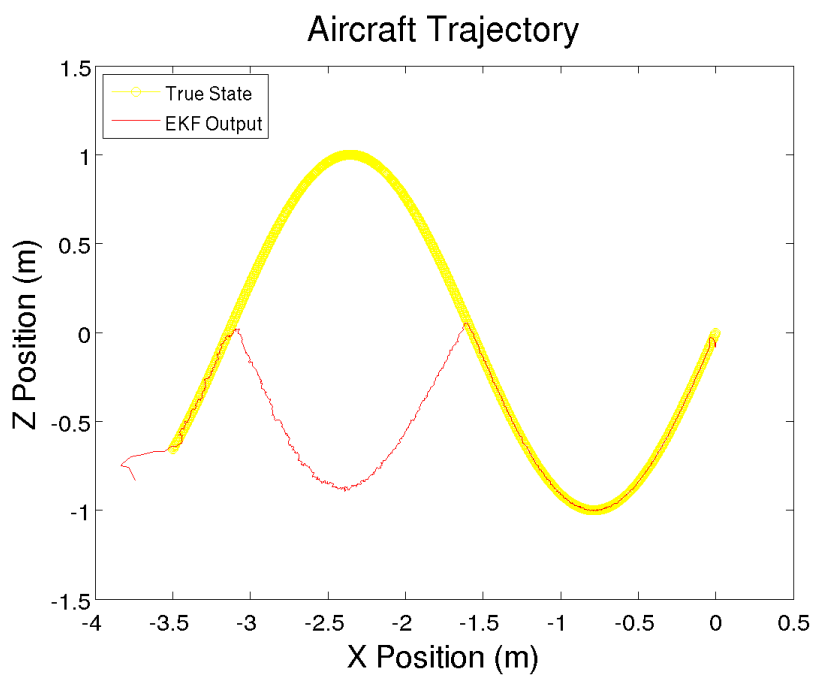

Fig. 12. Impoverished Model Extended Kalman Filter Simulation

to note that the state estimation failed as hypothesized at the zero-crossing due to the phase-amplitude ambiguity. However, to ensure this was the case, an Observability Analysis was carried out to ensure this failure was not due to linearization.

\section{B. Observability Analysis}

An Observability Analysis can provide valuable insight into the conditioning of the Kalman filter. A poorly conditioned observability matrix indicates that the extended Kalman filter may be inadequate to track a given system's states. The observability matrix can be constructed for each time $t$ as,

$$
\mathbf{O}=\left(\begin{array}{c}
H_{t} \\
H_{t} F_{t} \\
H_{t} F_{t}^{2} \\
H_{t} F_{t}^{n-1}
\end{array}\right)
$$

where $H_{t}$ is the measurement gradients matrix and $F_{t}$ is the process gradients matrix. If the rank of this matrix is ever less than the total number of states in the system, then the system is not observable. Occasionally, even though the matrix is still full rank, the difference between the largest and smallest eigenvalues/singular values is so great that the observability matrix will effectively loose rank. To better characterize this effect, the condition of the observability matrix was defined as follows:

$$
\operatorname{cond}(\overrightarrow{\mathbf{O}})=\frac{\lambda_{\max }}{\lambda_{\min }}
$$

The condition of the observability matrix was then plotted over the aircraft's trajectory, and is displayed in Figure 13. It is important to notice how the observability improves as the aircraft moves closer to the wire. This analysis does indicate that the system is not very observable far from the wire, however, it does not indicate that the EKF is failing due to linearizion or a singular measurement matrix.

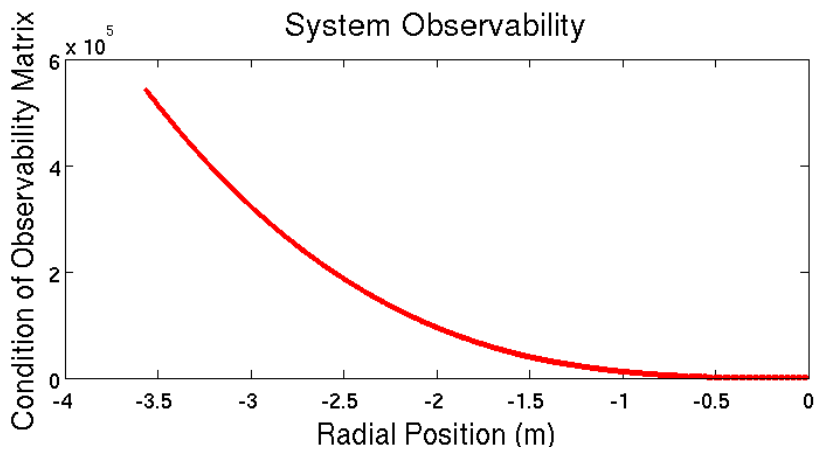

Fig. 13. Observability Analysis

\section{Complex Signal Tracking}

Since our observability analysis confirmed our suspicions about the impact of the field ambiguity, both the real and imaginary signal components were included in the state estimation algorithm to improve robustness. Notice that tracking through the field ambiguities is successful even when the double integrator model is used as demonstrated in Figures 14 and 15.

\section{Experiments}

With the simulations demonstrating promising results, a slow sweep was taken in the space around the power line, using on-board computation and electronics. These experimental results are shown in Figure 16. It can be observed that reasonable position estimates are obtained up to about 4 meters from the wire. As would be expected, because of the $\frac{1}{r^{3}}$ dependence of the field magnitude, the signal to noise ratio is much better closer to the wire. Once the state estimation was deemed satisfactory, the electronics were 


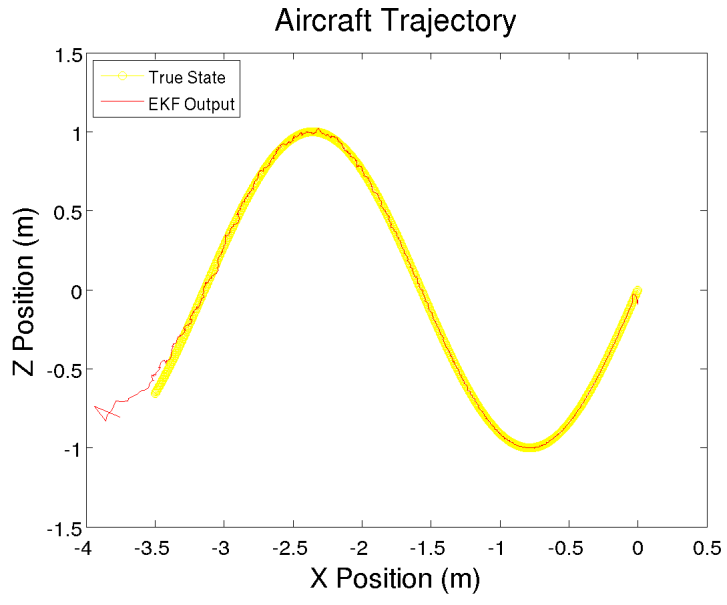

Fig. 14. Simulation of Extended Kalman Filter Tracking using Complex Measurements

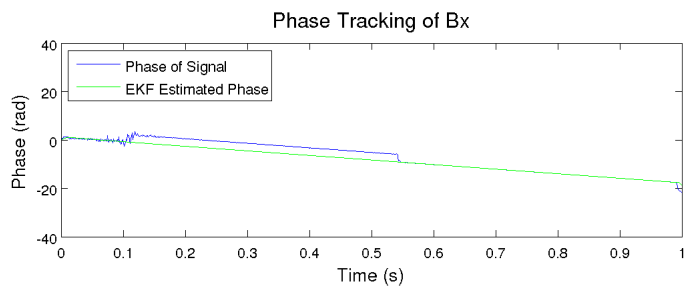

Phase Tracking of $\mathrm{Bz}$

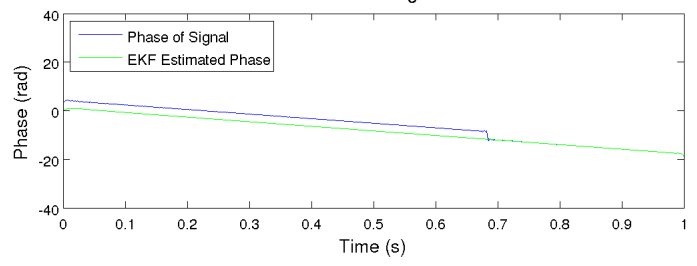

Fig. 15. Simulation of Extended Kalman Filter Phase Tracking

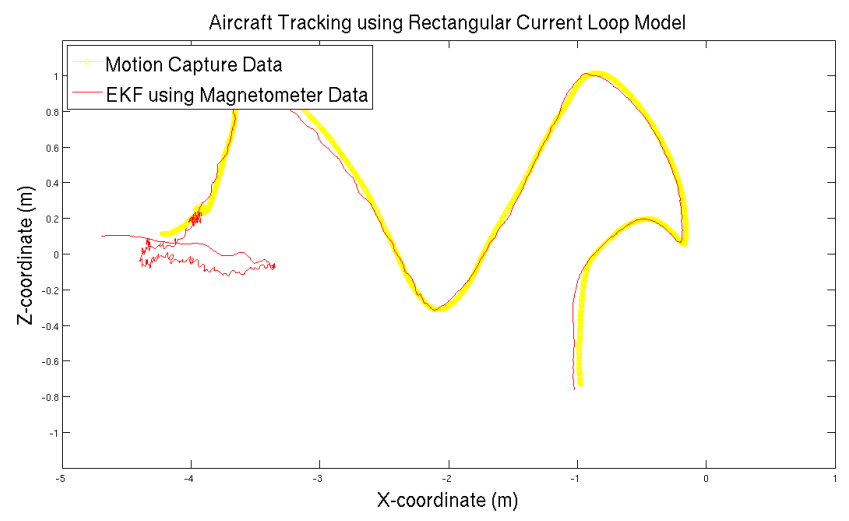

Fig. 16. Slow Sweep of Magnetic Field using Extended Kalman Filter

placed on board the aircraft, and the aircraft executed a nominal post-stall open-loop perching trajectory.

As seen in Figure 17 and 18, the state estimation demonstrated tracking with a reasonable accuracy up to $4 \mathrm{~m}$ from the wire.

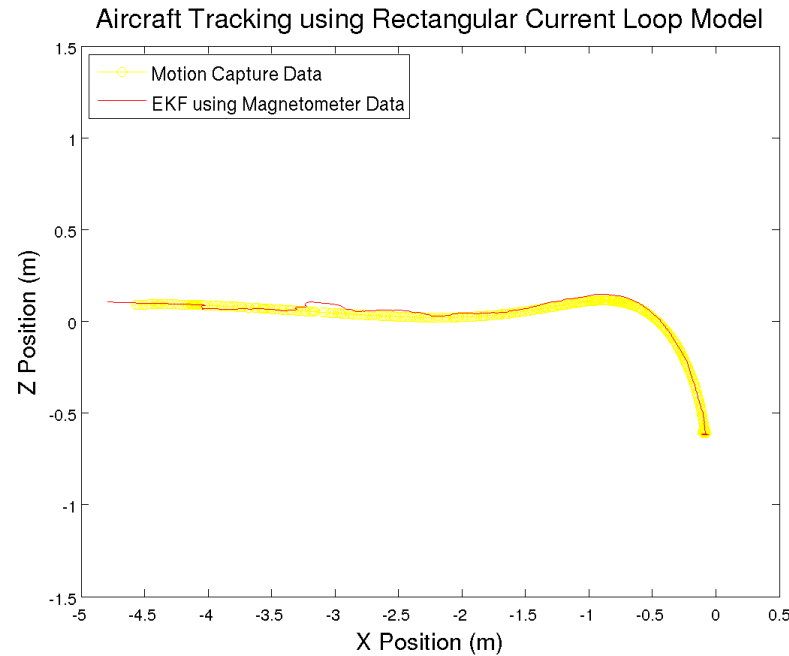

Fig. 17. Glider Tracking in Position
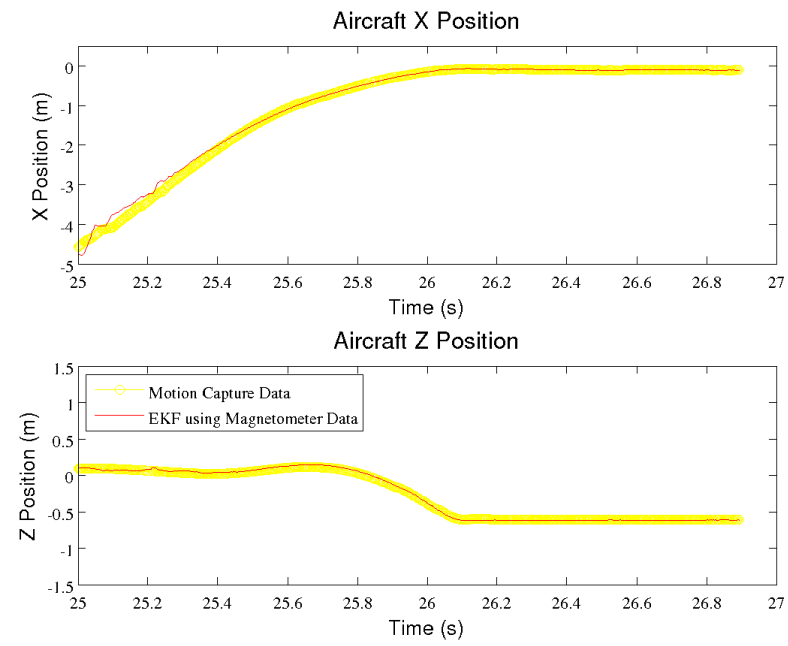

Fig. 18. Glider Tracking in Position and Time

\section{DISCUSSION}

As can be observed in the plots above, the magnetic field of a powerline can be used to effectively track an aircraft executing a perching maneuver with initial speeds of $8 \mathrm{~m} / \mathrm{s}$. The state estimates which are the output of the algorithm also seem to be comparable to many motion capture systems, exhibiting a worst case position error of $5 \mathrm{~cm}$ in $\mathrm{z}, 20 \mathrm{~cm}$ in $\mathrm{x}$, and a delay of about $13 \mathrm{~ms}$. It is also important to note that this methodology will easily extend to real world conditions, since it has the potential to be robust to both process and measurement models.

One could imagine that process model disturbances, such as wind gusts or aircraft model errors, could have a major impact on system performance if the state estimator relied heavily on aircraft dynamics. Fortunately, this estimation method, by using both the real and imaginary signal components, does not have to rely heavily on a knowledge of aircraft dynamics or initial conditions, as demonstrated in 
the use of the double integrator model.

In addition to being robust to the process model, the approach outlined in this paper can also be extended to account for deviations from the measurement model. First of all, it is important to realize that any polyphase system, such as three conductors carrying currents 180 degrees out of phase, will appear as a single phase signal at the magnetometer, just like the two-wire configuration in the experimental set up. However, unlike the experimental set-up, one would not know the current or the distance between the wires for a real powerline. However, if one did know the powerline configuration (3-wires, 2-wires, or 1-wire), one could easily estimate the current and distance parameters on-line. As was done with the states of the signal phase, the current and distance between wires could be incorporated into the state estimator as parameters that vary slowly with time. Although this would require an extra sensor to ensure that the system is observable, this extension would only involve a slight modification of the measurement equations.

\section{COnClusions And Future WORKS}

It can be concluded that by using the real and imaginary components output by the complex synchronous demodulation algorithm, and by writing the measurement model such that all magnetometer axes have the same phase, one can robustly track a perching aircraft with only a double integrator process model. Moreover, the system architecture which separates the demodulation process and the state estimation process also provides several other benefits. One benefit is that by allowing for the complex synchronous demodulation algorithm, which has low computational cost, to be executed at very high rates on a microcontroller or DSP, a significant oversampling can be achieved so as to improve measurement when signal to noise ratio is poor. Another benefit is that the state estimation algorithm, which can be quite computationally demanding, can be executed much more slowly, on the order of the aircraft dynamics, instead of being constrained to be $4 \mathrm{x}$ the carrier frequency.

In the future, the main goal of this work is to finally close the loop on the fixed-wing perching maneuver using magnetic field measurements. Another important future task is using the magnetic field sensing system in the vicinity of an DC brushless aircraft motor. As we transition to outdoor flight, a propeller will be crucial to rejecting wind gusts. Using a propeller will prove to be significantly challenging since the magnetic fields generated by DC motors will most certainly add interference. One solution to this problem is to build a model of the magnetic field generated by the DC motors and to add this in to the measurement equations of the state estimator.

\section{ACKNOWLEDGEMENTS}

This work was supported by the AFRL PLUS Flight Dynamics Testing program under subcontract MIT-1118 and DARPA's Young Faculty Award under award number N660001-09-1-2096.

\section{REFERENCES}

[1] Steven W. Blume. Electric Power System Basics. Institute of Electrical and Electronics Engineers, 2007.

[2] Theodore R. Bosela. Introduction to Electrical Power System Technology. Prentice-Hall, 1997.

[3] Rick Cory and Russ Tedrake. Experiments in fixed-wing UAV perching. In Proceedings of the AIAA Guidance, Navigation, and Control Conference. AIAA, 2008.

[4] G. Frulla. Preliminary reliability design of a solar-powered highaltitude very long endurance unmanned air vehicle. Journal of Aerospace Engineering, 216, 2002.

[5] Alberto Geri, Ambrogio Locatelli, and Giuseppe Maria Veca. Magnetic fields generated by power lines. Transactions on Magnetics, 31(3):1508-1511, May 1995.

[6] Adam Hadhazy. Air force invests in 'batman' technologies for special forces. TechNewsDaily, January 2011.

[7] Albert S. Huang, Edwin Olson, and David C. Moore. Lcm: Lightweight communications and marshalling. International Conference on Intelligent Robots and Systems (IROS), 2010 IEEE/RSJ, pages 4057-4062, October 2010.

[8] A.V. Mamishev and B.D. Russell. Measurement of magnetic fields in the direct proximity of power line conductors. IEEE Transactions of Power Delivery, 10(3):1211-1216, July 1995.

[9] Philippe Martin and Erwan Salaun. Invariant observers for attitude and heading estimation from low cost inertial and magnetic sensors. Proceedings of the IEEE on Decision and Control, December 2007.

[10] Joseph Moore and Russ Tedrake. Powerline perching with a fixed-wing UAV. In Proceedings of the AIAA Infotech@Aerospace Conference, Seattle, WA, April 2009. AIAA.

[11] A.V. Oppenheim, A.S. Willsky, and S. Hamid. Signals and Systems. Prentice Hall, 2nd edition, August 1996.

[12] Eric A. Prigge and Johnathan P. How. Signal architecture for a distributed magnetic local positioning system. IEEE Sensors Journal, 4(6), December 2004.

[13] Mark L. Psiaki, Francois Martel, and Parimal K. Pal. Three-axis attitude determination via kalman filtering of magnetometer data. Guidance, Navigation and Control, 13(3):506-514, May 1989.

[14] Kitt C. Reinhardt, Thomas R. Lamp, and Jack W. Geis. Solarpowered unmanned aerial vehicles. Energy Conversion Engineering Conference, 1996.

[15] John W. Roberts, Rick Cory, and Russ Tedrake. On the controllability of fixed-wing perching. In Proceedings of the American Controls Conference (ACC), 2009.

[16] J. J. Rodden and L. D. Montague. Design of an attitude control system with magnetometer sensors. American Institute of Aeronautics and Astronautics, 1(6):1422-1424, 1962.

[17] K. Sigurd and J. How. Uav trajectory design using total field collision avoidance. In AIAA Guidance, Navigation, and Control Conference and Exhibit, August 2003.

[18] Barry E. Tossman. Magnetic attitude control system for the radio astronomy explorer-a satellite. Spacecraft and Rockets, 6(3):239-244, 1969.

[19] John M. Wharington. Heuristic control of dynamic soaring. 5th Asian Control Conference, 2005.

[20] Adam M. Wickenheiser and Ephrahim Garcia. Longitudinal dynamics of a perching aircraft. Journal of Aircraft, 43(5):1386-1392, 2006.

[21] Adam M. Wickenheiser and Ephrahim Garcia. Optimization of perching maneuvers through vehicle morphing. Journal of Guidance, Control, and Dynamics, 31(4):815-824, July-August 2008. 